\title{
Estimating and Planning Accelerated Life Test Using Constant Stress for Generalized Logistic Distribution under Type-I Censoring
}

\author{
A. F. Attia, ${ }^{1}$ H. M. Aly, ${ }^{2}$ and S. O. Bleed ${ }^{1}$ \\ ${ }^{1}$ Department of Mathematical Statistics, Institute of Statistical Studies and Research, Cairo University, \\ Cairo 12613, Egypt \\ ${ }^{2}$ Department of Statistics, Faculty of Economics and Political Science, Cairo University, Cairo, Egypt
}

Correspondence should be addressed to H. M. Aly, han_m_hasan@yahoo.com

Received 24 September 2011; Accepted 16 October 2011

Academic Editors: F. Jauberteau, T. Y. Kam, and S. Sture

Copyright (C) 2011 A. F. Attia et al. This is an open access article distributed under the Creative Commons Attribution License, which permits unrestricted use, distribution, and reproduction in any medium, provided the original work is properly cited.

\begin{abstract}
The optimal designs and statistical inference of accelerated life tests under type-I are studied for constant stress-accelerated life tests (CSALTs). It is assumed that the lifetime at design stress has generalized logistic distribution. The scale parameter of the lifetime distribution at constant stress levels is assumed to be an inverse power law function of the stress level. The maximum likelihood (ML) estimators of the model parameters, Fisher information matrix, the asymptomatic variancecovariance matrix, the confidence bounds, the predictive value of the scale parameter, and the reliability function under the usual conditions are obtained under type-I censoring. Moreover, the optimal design of the accelerated life tests is studied according to the D-optimality criterion to specify the optimal censoring time. Finally, the numerical studies are introduced to illustrate the proposed procedures.
\end{abstract}

\section{Introduction}

Accelerated life testing (ALT) is becoming important and widely used in many fields (such as in manufacturing industries to assess or demonstrate component and subsystem reliability) because of rapidly changing technologies, higher customer expectations for better reliability, and the need for rapid device development. Briefly, ALT is a method for estimating the reliability of devices at normal use conditions from failure data obtained at severe conditions. The failure data is analyzed in terms of a suitable physical statistical model to obtain desired information about a device or its performance under normal use conditions.

Commonly, all available test data obtained from ALT is used in statistical analysis. However, the obtained data may be incomplete or it may include uncertainty about the failure 
time. There are three types of possible censoring schemes, right censoring, left censoring, and interval censoring. Moreover, the most common schemes are time-censoring and failurecensoring. Time-censored data is also known as type-I censored. It occurs when the life test is terminated at a specified time, before all units have failed. Data is failure censored (or type-II censored) if the test is terminated after a specified number of failures.

ALT can take the form of usage rate acceleration (for devices that do not operate continuously under normal conditions such as home appliances) or overstress acceleration (for devices with very high or continuous usage such as communication satellites, computers, and monitors). In real life, different types of stress loading may be considered when performing an accelerated test. The common types are constant stress, step stress, and progressive stress.

The most common stress loading is constant stress. In constant stress-accelerated life test (CSALT), the stress is kept at a constant level of stress throughout the life of the test; that is, each unit is run at a constant high stress level until the occurrence of failure or the observation is censored. Practically, most devices such as lamps, semiconductors, and microelectronics are run at a constant stress. Many authors have studied statistical inference of CSALT; for example, see Lawless [1], McCool [2], Bai and Chung [3], Bugaighis [4], Watkins [5], Abdel-Ghaly et al. [6], and El-Dessouky [7].

Before launching a new product, the manufacturer is always faced with decisions regarding the optimum method to estimate the reliability of the product or the service. Moreover, a test plan needs to be developed to obtain appropriate and sufficient information in order to accurately estimate the reliability performance at operating conditions, significantly reduce test times and costs, and achieve other objectives. The appropriate criteria for choosing a test plan depend on the purpose of the experiment (Meeker et al. [8]).

Optimum CSALT plans were studied for different lifetime distributions based on different censoring scheme; for example, Nelson and Kielpinski [9] studied optimum ALT plans for normal and lognormal life distributions. Nelson [10] reviewed statistically optimal and compromise plans for the single stress ALT planning problem. Yang [11] proposed an optimal design of 4-level constant-stress ALT plans considering different censoring times. Ding et al. [12] dealt with Weibull distribution.

The term Generalized Logistic (GL) distribution is used as the name for several different families of probability distributions (see Johnson et al. [13]). The main feature of the GL distribution is that new parameters were introduced to control both location and scale. It allows for a greater degree of flexibility and it is expected to be useful in many more practical situations (Nadarajah and Kotz [14]), for example, in extreme value event evaluation, in hydrological risk analysis, and in a quanta response data, and to model the data with a unimodal density (for more details, see Mathai and Provost [15], Tolikas [16], Alkasasbeh and Raqab [17], Tolikas and Gettinby [18], Shabri et al. [19], and Tolikas [20]).

Alkasasbeh and Raqab [17] and Shin et al. [21] were interested in estimating the parameters of the GL distribution by ML and other methods while Rai et al. [22] employed the genetic algorithm to derive the unit hydrograph by using nine distributions including GL distribution. Kim et al. [23] derived the probability plot correlation coefficient test statistic for the GL distribution. Shabri et al. [19] introduced the method of trimmed L-moments as an alternative way in estimating the flood for higher return period and derived the trimmed L-moments for the GL distribution. However, no studies have been made on estimating or planning (optimal design) CSALT using the GL distribution.

This paper is organized as follows. In Section 2, the underlying distribution and the test method are described. Section 3 introduces the ML estimators of the model parameters with their properties under type-I censoring. Optimum censoring time test plan is developed 
in Section 4. Finally, the simulation studies needed for illustrating the theoretical results are presented in Section 5.

\section{The Model}

\subsection{The Generalized Logistic Distribution}

The probability density function (pdf) of a three-parameter-generalized logistic distribution (Molenberghs and Verbeke [24]) is given by

$$
f(x)=\alpha \gamma e^{\alpha x}\left(1+\frac{\gamma}{\theta} e^{\alpha x}\right)^{-(\theta+1)}, \quad-\infty<x<\infty, \alpha, \gamma, \theta>0 .
$$

The reliability function takes the form

$$
R(x)=\left(1+\frac{\gamma}{\theta} e^{\alpha x}\right)^{-\theta}, \quad-\infty<x<\infty, \alpha, \gamma, \theta>0
$$

and the corresponding failure rate is given by

$$
h(x)=\alpha \gamma e^{\alpha x}\left(1+\frac{\gamma}{\theta} e^{\alpha x}\right)^{-1}, \quad-\infty<x<\infty, \alpha, \gamma, \theta>0
$$

\subsection{Assumptions}

We assume the following assumptions for the CSALT procedure.

(i) A total of $N$ units are divided into $n_{1}, n_{2}, \ldots, n_{k}$ units where $\sum_{j=1}^{k} n_{j}=N$.

(ii) There are $k$ levels of high stress $V_{j}, j=1, \ldots, k$ in the experiment, and $V_{u}$ is the stress under usual conditions, where $V_{u}<V_{1}<\cdots<V_{k}$.

(iii) Each $n_{j}, j=1, \ldots, k$ unit in the experiment is run at a prespecified constant stress $V_{j}, j=1, \ldots, k$.

(iv) It is assumed that the stress affected only on the scale parameter of the underlying distribution.

(v) The failure times $x_{i j}, i=1, \ldots, n_{j}$ and $j=1, \ldots, k$, at stress levels $V_{j}, j=$ $1, \ldots, k$, are the 3 -parameter-generalized logistic distribution with probability density function:

$$
\begin{array}{r}
f\left(x_{i j}, \alpha_{j}, \gamma, \theta\right)=\alpha_{j} \gamma e^{\alpha_{j} x_{i j}}\left(1+\frac{\gamma}{\theta} e^{\alpha_{j} x_{i j}}\right)^{-(\theta+1)}, \\
-\infty<x_{i j}<\infty, \quad \alpha_{j}, \gamma, \theta>0, \quad i=1, \ldots, n_{j}, \quad j=1, \ldots, k .
\end{array}
$$


(vi) The scale parameter $\alpha_{j}, j=1, \ldots, k$, of the underlying lifetime distribution (2.4) is assumed to have an inverse power law function on stress levels, that is,

$$
\begin{gathered}
\alpha_{j}=C S_{j}^{P}, \quad C, P>0, \quad \text { where } S_{j}=\frac{V^{*}}{V_{j}}, \\
V^{*}=\prod_{j=1}^{k} V_{j}^{b_{j}}, \quad b_{j}=\frac{n_{j}}{\sum_{j=1}^{k} n_{j}},
\end{gathered}
$$

where $C$ is the constant of proportionality, and $P$ is the power of the applied stress.

\section{Maximum Likelihood (ML) Estimation}

In addition to the common assumptions in Section 2.2, we assume that the experiment is terminated at a prespecified censoring time $T_{j}$. Thus, the corresponding likelihood function will be as in the following form:

$$
L=\prod_{j=1}^{k} \prod_{i=1}^{n_{j}}\left[C S_{j}^{P} \gamma e^{C S_{j}^{P} x_{i j}}\left(1+\frac{\gamma}{\theta} e^{C S_{j}^{P} x_{i j}}\right)^{-(\theta+1)}\right]^{\delta_{i j}}\left[\left(1+\frac{\gamma}{\theta} e^{C S_{j}^{P} T_{j}}\right)^{-\theta}\right]^{1-\delta_{i j}}
$$

where $\delta_{i j}$ is an indicator variable, such that

$$
\delta_{i j}=\left\{\begin{array}{lll}
1 & \text { if } & x_{i j} \leq T_{j} \\
0 & \text { if } & x_{i j}>T_{j}
\end{array}\right.
$$

The log-likelihood function is

$$
\begin{aligned}
\ln L= & \ln C \sum_{j=1}^{k} \sum_{i=1}^{n_{j}} \delta_{i j}+P \sum_{j=1}^{k} \sum_{i=1}^{n_{j}} \delta_{i j} \ln S_{j}+\ln \gamma \sum_{j=1}^{k} \sum_{i=1}^{n_{j}} \delta_{i j}+C \sum_{j=1}^{k} \sum_{i=1}^{n_{j}} \delta_{i j} S_{j}^{P} x_{i j} \\
& -(\theta+1) \sum_{j=1}^{k} \sum_{i=1}^{n_{j}} \delta_{i j} \ln \left(1+\frac{\gamma}{\theta} e^{C S_{j}^{P} x_{i j}}\right)-\theta \sum_{j=1}^{k} \sum_{i=1}^{n_{j}}\left(1-\delta_{i j}\right) \ln \left(1+\frac{\gamma}{\theta} e^{C S_{j}^{P} T_{j}}\right)
\end{aligned}
$$

\subsection{Estimation of the Parameters}

The first derivatives of the log-likelihood function (3.3) with respect to the unknown parameters $C, P, \gamma$, and $\theta$ are

$$
\begin{aligned}
& \frac{\partial \ln L}{\partial C}=C^{-1} \sum_{j=1}^{k} \sum_{i=1}^{n_{j}} \delta_{i j}+\sum_{j=1}^{k} \sum_{i=1}^{n_{j}} \delta_{i j} S_{j}^{P} x_{i j}-(\theta+1) \sum_{j=1}^{k} \sum_{i=1}^{n_{j}} \delta_{i j} \xi_{i j}-\theta \sum_{j=1}^{k} \sum_{i=1}^{n_{j}}\left(1-\delta_{i j}\right) Z_{j}, \\
& \frac{\partial \ln L}{\partial P}=\sum_{j=1}^{k} \sum_{i=1}^{n_{j}} \delta_{i j} \ln S_{j}+C\left(\sum_{j=1}^{k} \sum_{i=1}^{n_{j}} \delta_{i j} \sigma_{i j}-(\theta+1) \sum_{j=1}^{k} \sum_{i=1}^{n_{j}} \delta_{i j} \ln S_{j} \xi_{i j}-\theta \sum_{j=1}^{k} \sum_{i=1}^{n_{j}}\left(1-\delta_{i j}\right) \ln S_{j} Z_{j}\right),
\end{aligned}
$$




$$
\begin{aligned}
& \frac{\partial \ln L}{\partial \gamma}=\frac{1}{\gamma}\left\{\sum_{j=1}^{k} \sum_{i=1}^{n_{j}} \delta_{i j}-(\theta+1) \sum_{j=1}^{k} \sum_{i=1}^{n_{j}} \delta_{i j} v_{i j}-\theta \sum_{j=1}^{k} \sum_{i=1}^{n_{j}}\left(1-\delta_{i j}\right) \mu_{j}\right\}, \\
& \frac{\partial \ln L}{\partial \theta}=\sum_{j=1}^{k} \sum_{i=1}^{n_{j}} \delta_{i j}\left(\frac{\theta+1}{\theta} v_{i j}-\pi_{i j}\right)+\sum_{j=1}^{k} \sum_{i=1}^{n_{j}}\left(1-\delta_{i j}\right)\left(\mu_{j}-\Lambda_{j}\right),
\end{aligned}
$$

where $\xi_{i j}=s_{j}^{P} x_{i j} v_{i j}, v_{i j}=\left(1+(\theta / \gamma) e^{-C S_{j}^{P} x_{i j}}\right)^{-1}, Z_{j}=S_{j}^{P} T_{j} \mu_{j}, \mu_{j}=\left(1+(\theta / \gamma) e^{-C S_{j}^{P} T_{j}}\right)^{-1}, \pi_{i j}=$ $-\ln \left(1-v_{i j}\right), \Lambda_{j}=-\ln \left(1-\mu_{j}\right)$, and $\sigma_{i j}=S_{j}^{P} x_{i j} \ln S_{j}$.

Since the first derivative equations (3.4) are nonlinear equations, their solutions will be obtained numerically by using the Math-Cade program as will be seen in Section 5.1. The second partial derivatives of the log-likelihood function (3.3) with respect to the parameters $C, P, \gamma$, and $\theta$ are as follows:

$$
\begin{aligned}
& \frac{\partial^{2} \ln L}{\partial C^{2}}=\frac{-1}{r}\left\{\frac{r \sum_{j=1}^{k} \sum_{i=1}^{n_{j}} \delta_{i j}}{C^{2}}+\theta(\theta+1) \sum_{j=1}^{k} \sum_{i=1}^{n_{j}} \delta_{i j} \xi_{i j}^{2} e^{-C S_{j}^{P} x_{i j}}+\theta^{2} \sum_{j=1}^{k} \sum_{i=1}^{n_{j}}\left(1-\delta_{i j}\right) Z_{j}^{2} e^{-C S_{j}^{P} T_{j}}\right\}, \\
& \frac{\partial^{2} \ln L}{\partial P^{2}}=-C\left\{\sum_{j=1}^{\mathrm{k}} \sum_{i=1}^{n_{j}} \delta_{i j} \ln S_{j}\left[\sigma_{i j}\left(C(\theta+1) \xi_{i j}\left(1-v_{i j}\right)-1\right)+(\theta+1) \xi_{i j} \ln S_{j}\right]\right. \\
& \left.+\theta \sum_{j=1}^{k} \sum_{i=1}^{n_{j}}\left(1-\delta_{i j}\right) Z_{j} \ln S_{j}\left(C \Omega_{j}\left(1-\mu_{j}\right)+\ln S_{j}\right)\right\}, \\
& \frac{\partial^{2} \ln L}{\partial \gamma^{2}}=\frac{-1}{\gamma^{2}}\left\{\sum_{j=1}^{k} \sum_{i=1}^{n_{j}} \delta_{i j}-(\theta+1) \sum_{j=1}^{k} \sum_{i=1}^{n_{j}} \delta_{i j} \nu_{i j}^{2}-\theta \sum_{j=1}^{k} \sum_{i=1}^{n_{j}}\left(1-\delta_{i j}\right) \mu_{j}^{2}\right\}, \\
& \frac{\partial^{2} \ln L}{\partial \theta^{2}}=\frac{-1}{\theta}\left\{\frac{1}{\theta} \sum_{j=1}^{k} \sum_{i=1}^{n_{j}} \delta_{i j} \nu_{i j}\left[(1-\theta)+(1+\theta)\left(1-v_{i j}\right)\right]-\sum_{j=1}^{k} \sum_{i=1}^{n_{j}}\left(1-\delta_{i j}\right) \mu_{j}^{2}\right\}, \\
& \frac{\partial^{2} \ln L}{\partial C \partial P}=-\left\{\sum_{j=1}^{k} \sum_{i=1}^{n_{j}} \delta_{i j}\left[(\theta+1) \xi_{i j}\left(C \sigma_{i j}\left(1-v_{i j}\right)+\ln S_{j}\right)-\sigma_{i j}\right]\right. \\
& \left.+\theta \sum_{j=1}^{k} \sum_{i=1}^{n_{j}}\left(1-\delta_{i j}\right) Z_{j}\left(C \Omega_{j}\left(1-\mu_{j}\right)+\ln S_{j}\right)\right\}, \\
& \frac{\partial^{2} \ln L}{\partial C \partial \gamma}=\frac{-1}{\gamma}\left\{(\theta+1) \sum_{j=1}^{k} \sum_{i=1}^{n_{j}} \delta_{i j} \xi_{i j}\left(1-v_{i j}\right)+\theta \sum_{j=1}^{k} \sum_{i=1}^{n_{j}}\left(1-\delta_{i j}\right) Z_{j}\left(1-\mu_{j}\right)\right\}, \\
& \frac{\partial^{2} \ln L}{\partial C \partial \theta}=-\left\{\sum_{j=1}^{k} \sum_{i=1}^{n_{j}} \delta_{i j} \xi_{i j}\left(1-\frac{(\theta+1)}{\theta}\left(1-v_{i j}\right)\right)+\sum_{j=1}^{k} \sum_{i=1}^{n_{j}}\left(1-\delta_{i j}\right) \mu_{j} Z_{j}\right\},
\end{aligned}
$$




$$
\begin{aligned}
& \frac{\partial^{2} \ln L}{\partial P \partial \gamma}=\frac{-C}{\gamma}\left\{(\theta+1) \sum_{j=1}^{k} \sum_{i=1}^{n_{j}} \delta_{i j} \xi_{i j}\left(1-v_{i j}\right) \ln S_{j}+\theta \sum_{j=1}^{k} \sum_{i=1}^{n_{j}}\left(1-\delta_{i j}\right) Z_{j}\left(1-\mu_{j}\right) \ln S_{j}\right\}, \\
& \frac{\partial^{2} \ln L}{\partial p \partial \theta}=-C\left\{\sum_{j=1}^{k} \sum_{i=1}^{n_{j}} \delta_{i j} \xi_{i j} \ln S_{j}\left[1-\left(1+\theta^{-1}\right)\left(1-v_{i j}\right)\right]+\sum_{j=1}^{k} \sum_{i=1}^{n_{j}}\left(1-\delta_{i j}\right) \mu_{j} Z_{j} \ln S_{j}\right\}, \\
& \frac{\partial^{2} \ln L}{\partial \gamma \partial \theta}=\frac{-1}{\gamma}\left\{\sum_{j=1}^{k} \sum_{i=1}^{n_{j}} \delta_{i j} \nu_{i j}\left[1-\left(1+\theta^{-1}\right)\left(1-v_{i j}\right)\right]+\sum_{j=1}^{k} \sum_{i=1}^{n_{j}}\left(1-\delta_{i j}\right) \mu_{j}^{2}\right\},
\end{aligned}
$$

where $\Omega_{j}=S_{j}^{P} T_{j} \ln S_{j}$.

Therefore, the elements of the Fisher information matrix for the MLE can be obtained as the expectations of the negative of the second partial derivatives, that is,

$$
F=\left(\begin{array}{rrrr}
f_{11} & f_{12} & f_{13} & f_{14} \\
f_{22} & f_{23} & f_{24} \\
& f_{33} & f_{34} \\
& & & f_{44}
\end{array}\right)=-E\left(\begin{array}{cccc}
\frac{\partial^{2} \ln L}{\partial c^{2}} & \frac{\partial^{2} \ln L}{\partial c \partial p} & \frac{\partial^{2} \ln L}{\partial c \partial \gamma} & \frac{\partial^{2} \ln L}{\partial c \partial \theta} \\
& \frac{\partial^{2} \ln L}{\partial p^{2}} & \frac{\partial^{2} \ln L}{\partial p \partial \gamma} & \frac{\partial^{2} \ln L}{\partial p \partial \theta} \\
& & \frac{\partial^{2} \ln L}{\partial \gamma^{2}} & \frac{\partial^{2} \ln L}{\partial \gamma \partial \theta} \\
& & & \frac{\partial^{2} \ln L}{\partial \theta^{2}}
\end{array}\right) .
$$

The asymptotic variance-covariance matrix for the MLE is defined as the inverse of the Fisher information matrix (3.6), that is,

$$
\Sigma=\widehat{F}^{-1}
$$

\subsection{Prediction of the Scale Parameter and the Reliability Function}

To predict the value of the scale parameter $\alpha_{u}$ under the usual condition stress $V_{u}$, the invariance property of MLE is used (for more details see Meeker et al. [8]), that is,

$$
\begin{gathered}
\widehat{\alpha}=\widehat{C} S_{u}^{\widehat{P}}, \quad \text { where } S_{u}=\frac{V^{*}}{V_{u}}, \\
V^{*}=\prod_{j=1}^{k} V_{j}^{b_{j}}, \quad b_{j}=\frac{n_{j}}{\sum_{j=1}^{k} n_{j}} .
\end{gathered}
$$

The MLE of the reliability function at the lifetime $x_{0}$ under the usual condition stress $V_{u}$, is given by

$$
\widehat{R}_{u}\left(x_{0}\right)=\left(1+\frac{\gamma}{\theta} e^{\widehat{\alpha}_{u} x_{0}}\right)^{-\theta} .
$$




\section{Optimum Test Plan}

Before starting an accelerated life test (which is sometimes an expensive and difficult endeavor), it is advisable to have a plan that helps in accurately estimating reliability at operating conditions while minimizing test time and cost.

In this section, we determine the best choice of the values of the censoring times $T_{j}, j=$ $1, \ldots, k$, according to the D-Optimality criterion. The criterion is based on the minimization of the determinant of Fisher information matrix of the MLE of the model parameters (Gouno [25]). Therefore, the optimal value of $T_{j}$ at each stress level $V_{j}, j=1, \ldots, k$, can be obtained by solving the following equation:

$$
\frac{\partial|F|}{\partial T_{j}}=0, \quad j=1, \ldots, k
$$

The determinant of $F$ and the derivation of (4.1) are placed in the appendix. To get the optimum values of the stress change time $T_{1}$ and $T_{2}$ that minimized $|F|$ of the MLE under the stress level $V_{j}, j=1,2$, the numerical solution is obtained as will be shown in Section 5.2.

\section{Simulation Studies}

This section presents the numerical solutions to obtain the MLE of the unknown parameters $C, P, \gamma$, and $\theta$, their mean squared errors (MSEs), relative absolute biases (RAB), Lower Bound (LB), Upper Bound (UB), the estimated of scale parameter $\alpha$, and reliability function $R\left(x_{0}\right)$ under normal use conditions $V_{u}$. Also, it presents the numerical solutions to determine the best choice values of the stress change time $T_{1}^{*}$ and $T_{2}^{*}$.

\subsection{MLE under Type-I Censoring}

In this section, the numerical solution is performed according to the following steps.

(i) For given values of $C, P$, and stress level $V_{j}, j=1,2,3$, the values of $\alpha_{j}, j=1,2,3$ are calculated according to (2.5).

(ii) Generate a random sample of size $N$ from the 3-parameter-generalized logistic distribution and obtain the random variables $x_{i j},\left\{i=1, \ldots, n_{j}, j=1, \ldots, k\right\}$ for given values of $n_{j}, T_{j}, j=1, \ldots, k$, and different initial guesses of the true parameters $\alpha, \gamma, \theta$, say $\alpha_{0}, \gamma_{0}, \theta_{0}$.

(iii) Based on the values of $n_{j}, T_{j}, V_{j}, x_{i j},\left\{i=1, \ldots, n_{j}, j=1, \ldots, k\right\}$, and $V_{u}$, the MLE and their MSE, RAB, LB, and UB, in addition to $\widehat{\alpha}_{u}$ and $\widehat{R}_{u}\left(x_{0}\right)$, are obtained.

(iv) The steps are repeated more than 150 times until getting the MLE as shown in Table 1.

The numerical results which are placed in Tables 1 to 4 are based on $n_{1}=29, n_{2}=10, n_{3}=2$, $T_{1}=4, T_{2}=3, T_{3}=2, V_{1}=0.75, V_{2}=1.5, V_{3}=2.25$, and $V_{u}=0.5$.

From the results of Tables 1, 2, 3 and 4, we observe that the MSE of the scale parameter $\alpha_{j}, j=1,2,3$, decreases as the stress value $V_{j}, j=1,2,3$, increases. In general, the MSE decrease as the values of $C_{0}$ and $\gamma_{0}$ decreases at the same values of $P_{0}$ and $\theta_{0}$, while in the variancecovariance matrix we observe that the covariance between $C$ and $P$ is the smallest one and converges to zero. The reliability decrease when the mission time $x_{0}$ increases. It is reduced when the values of $C_{0}$ decrease and the values of $P_{0}, \gamma_{0}$, and $\theta_{0}$ increase at the same mission time. 
Table 1: The MLE, RAB, and MSE.

\begin{tabular}{|c|c|c|c|c|c|c|c|}
\hline$C_{0}$ & $P_{0}$ & $\gamma_{0}$ & $\theta_{0}$ & Parameter & MLE & RAB & MSE \\
\hline \multirow{7}{*}{1.0} & \multirow{7}{*}{1.0} & \multirow{7}{*}{1.25} & \multirow{7}{*}{0.7} & C & 1.10902 & 0.10902 & 0.01189 \\
\hline & & & & $P$ & 0.98314 & 0.01686 & 0.00028 \\
\hline & & & & $r$ & 1.22511 & 0.01991 & 0.00062 \\
\hline & & & & $\theta$ & 0.88398 & 0.11602 & 0.01346 \\
\hline & & & & $\alpha_{1}$ & 1.38040 & 0.10486 & 0.01717 \\
\hline & & & & $\alpha_{2}$ & 0.69831 & 0.11785 & 0.00542 \\
\hline & & & & $\alpha_{3}$ & 0.46874 & 0.12552 & 0.00273 \\
\hline \multirow{7}{*}{1.0} & \multirow{7}{*}{1.0} & \multirow{7}{*}{1.3} & \multirow{7}{*}{1.0} & C & 1.15695 & 0.15695 & 0.02463 \\
\hline & & & & $P$ & 0.9846 & 0.0154 & 0.00024 \\
\hline & & & & $r$ & 1.23098 & 0.05309 & 0.00476 \\
\hline & & & & $\theta$ & 0.87707 & 0.12293 & 0.12446 \\
\hline & & & & $\alpha_{1}$ & 1.44053 & 0.15299 & 0.03654 \\
\hline & & & & $\alpha_{2}$ & 0.72799 & 0.16536 & 0.01067 \\
\hline & & & & $\alpha_{3}$ & 0.48837 & 0.17266 & 0.00517 \\
\hline \multirow{7}{*}{1.0} & \multirow{7}{*}{1.0} & \multirow{7}{*}{1.5} & \multirow{7}{*}{1.0} & $C$ & 1.35169 & 0.35169 & 0.12369 \\
\hline & & & & $P$ & 0.99012 & 0.00988 & 0.00010 \\
\hline & & & & $r$ & 1.25504 & 0.16331 & 0.06001 \\
\hline & & & & $\theta$ & 0.84992 & 0.15008 & 0.02252 \\
\hline & & & & $\alpha_{1}$ & 1.68507 & 0.34872 & 0.18982 \\
\hline & & & & $\alpha_{2}$ & 0.84832 & 0.35799 & 0.05001 \\
\hline & & & & $\alpha_{3}$ & 0.56782 & 0.36344 & 0.02291 \\
\hline \multirow{7}{*}{1.0} & \multirow{7}{*}{1.1} & \multirow{7}{*}{1.4} & \multirow{7}{*}{1.0} & C & 1.26473 & 0.26473 & 0.07008 \\
\hline & & & & $P$ & 0.97595 & 0.11277 & 0.01539 \\
\hline & & & & $r$ & 1.37052 & 0.02106 & 0.00087 \\
\hline & & & & $\theta$ & 0.8474 & 0.1526 & 0.02329 \\
\hline & & & & $\alpha_{1}$ & 1.5717 & 0.23028 & 0.08654 \\
\hline & & & & $\alpha_{2}$ & 0.79906 & 0.34074 & 0.04124 \\
\hline & & & & $\alpha_{3}$ & 0.53793 & 0.4099 & 0.02446 \\
\hline \multirow{7}{*}{1.25} & \multirow{7}{*}{1.1} & \multirow{7}{*}{1.25} & \multirow{7}{*}{1.0} & $C$ & 1.14000 & 0.08800 & 0.01210 \\
\hline & & & & $P$ & 0.95459 & 0.13219 & 0.02114 \\
\hline & & & & $r$ & 1.37506 & 0.10005 & 0.01564 \\
\hline & & & & $\theta$ & 1.01030 & 0.01030 & 0.00011 \\
\hline & & & & $\alpha_{1}$ & 1.40997 & 0.11705 & 0.03494 \\
\hline & & & & $\alpha_{2}$ & 0.72753 & 0.02342 & 0.00030 \\
\hline & & & & $\alpha_{3}$ & 0.49403 & 0.03589 & 0.00029 \\
\hline \multirow{7}{*}{1.4} & \multirow{7}{*}{1.0} & \multirow{7}{*}{1.0} & \multirow{7}{*}{0.7} & C & 0.91213 & 0.34848 & 0.23802 \\
\hline & & & & $P$ & 0.68352 & 0.31648 & 0.10016 \\
\hline & & & & $r$ & 1.25991 & 0.25991 & 0.06755 \\
\hline & & & & $\theta$ & 1.10442 & 0.57774 & 0.16356 \\
\hline & & & & $\alpha_{1}$ & 1.06206 & 0.39281 & 0.47207 \\
\hline & & & & $\alpha_{2}$ & 0.66129 & 0.24387 & 0.04549 \\
\hline & & & & $\alpha_{3}$ & 0.50122 & 0.14034 & 0.00670 \\
\hline
\end{tabular}


Table 1: Continued.

\begin{tabular}{cccccccc}
\hline$C_{0}$ & $P_{0}$ & $\gamma_{0}$ & $\theta_{0}$ & Parameter & MLE & RAB & MSE \\
\hline & & & $C$ & 0.91672 & 0.3452 & 0.23356 \\
& & & $P$ & 0.84089 & 0.29926 & 0.12896 \\
& & & & $r$ & 1.49253 & 0.49253 & 0.24259 \\
1.4 & 1.2 & \multirow{2}{*}{0.9} & $\theta$ & 1.10045 & 0.22272 & 0.04018 \\
& & & $\alpha_{1}$ & 1.10547 & 0.39552 & 0.52319 \\
& & & $\alpha_{2}$ & 0.61718 & 0.22467 & 0.03198 \\
& & & $\alpha_{3}$ & 0.43888 & 0.10314 & 0.00255 \\
\hline
\end{tabular}

Table 2: The confidence intervals.

\begin{tabular}{|c|c|c|c|c|c|c|c|}
\hline$C_{0}$ & $P_{0}$ & $\gamma_{0}$ & $\theta_{0}$ & Parameter & Variance & L.B & U.B \\
\hline \multirow{4}{*}{1.0} & \multirow{4}{*}{1.0} & \multirow{4}{*}{1.25} & \multirow{4}{*}{0.7} & C & 0.05771 & 0.79600 & 1.42204 \\
\hline & & & & $P$ & 0.21348 & 0.38110 & 1.58518 \\
\hline & & & & $r$ & 0.32283 & 0.48477 & 1.96545 \\
\hline & & & & $\theta$ & 0.35710 & 0.10533 & 1.66263 \\
\hline \multirow{4}{*}{1.0} & \multirow{4}{*}{1.0} & \multirow{4}{*}{1.3} & \multirow{4}{*}{1.0} & C & 0.05362 & 0.85523 & 1.45867 \\
\hline & & & & $P$ & 0.22330 & 0.36887 & 1.60033 \\
\hline & & & & $\gamma$ & 0.36304 & 0.44588 & 2.01608 \\
\hline & & & & $\theta$ & 0.33362 & 0.12446 & 1.62968 \\
\hline \multirow{4}{*}{1.0} & \multirow{4}{*}{1.0} & \multirow{4}{*}{1.5} & \multirow{4}{*}{1.0} & $C$ & 0.05225 & 1.05385 & 1.64953 \\
\hline & & & & $P$ & 0.26782 & 0.31580 & 1.66444 \\
\hline & & & & $r$ & 0.74628 & 0.12942 & 2.38066 \\
\hline & & & & $\theta$ & 0.36300 & 0.06488 & 1.63496 \\
\hline \multirow{4}{*}{1.0} & \multirow{4}{*}{1.1} & \multirow{4}{*}{1.4} & \multirow{4}{*}{1.0} & C & 0.03979 & 1.00482 & 1.52464 \\
\hline & & & & $P$ & 0.25300 & 0.32055 & 1.63135 \\
\hline & & & & $r$ & 0.32636 & 0.62614 & 2.11490 \\
\hline & & & & $\theta$ & 0.22237 & 0.23296 & 1.46184 \\
\hline \multirow{4}{*}{1.25} & \multirow{4}{*}{1.1} & \multirow{4}{*}{1.25} & \multirow{4}{*}{1.0} & $C$ & 0.10822 & 0.71135 & 1.56865 \\
\hline & & & & $P$ & 0.17555 & 0.40865 & 1.50053 \\
\hline & & & & $\gamma$ & 0.28023 & 0.68529 & 2.06483 \\
\hline & & & & $\theta$ & 0.42254 & 0.16331 & 1.85729 \\
\hline \multirow{4}{*}{1.4} & \multirow{4}{*}{1.0} & \multirow{4}{*}{1.0} & \multirow{4}{*}{0.7} & C & 0.09833 & 0.50354 & 1.32072 \\
\hline & & & & $P$ & 0.15330 & 0.17334 & 1.19370 \\
\hline & & & & $r$ & 0.09398 & 0.86046 & 1.65936 \\
\hline & & & & $\theta$ & 0.09605 & 0.70059 & 1.50825 \\
\hline \multirow{4}{*}{1.4} & \multirow{4}{*}{1.2} & \multirow{4}{*}{1.0} & \multirow{4}{*}{0.9} & $C$ & 0.25885 & 0.25379 & 1.57965 \\
\hline & & & & $P$ & 0.14056 & 0.35238 & 1.32940 \\
\hline & & & & $\gamma$ & 0.11485 & 1.00334 & 1.98172 \\
\hline & & & & $\theta$ & 0.37572 & 0.01707 & 1.99193 \\
\hline
\end{tabular}

\subsection{Optimum Test Plan of Censoring Times}

In order to obtain the optimum values of the censoring times $T_{1}$ and $T_{2}$, a numerical solution is performed by generating a random sample of size $N$ from the 3-parameter-generalized logistic distribution. The random variables $x_{i j}\left\{i=1, \ldots, n_{j}, j=1,2\right\}$ for given values of $n_{j}$, $T_{j}, j=1,2$, and different values of $\left(C_{0}, P_{0}, \gamma_{0}, \theta_{0}\right)$ are obtained. Therefore, the optimum values 
Table 3: The asymptotic variance-covariance matrix.

\begin{tabular}{|c|c|c|c|c|c|c|c|c|}
\hline$C_{0}$ & $P_{0}$ & $r_{0}$ & $\theta_{0}$ & Parameter & $C$ & $P$ & $r$ & $\theta$ \\
\hline \multirow{4}{*}{1.0} & \multirow{4}{*}{1.0} & \multirow{4}{*}{1.25} & \multirow{4}{*}{0.7} & C & 0.05771 & 0.00595 & 0.09077 & -0.117570 \\
\hline & & & & $P$ & & 0.21348 & 0.04473 & -0.05503 \\
\hline & & & & $\gamma$ & & & 0.32283 & -0.26978 \\
\hline & & & & $\theta$ & & & & 0.35710 \\
\hline \multirow{4}{*}{1.0} & \multirow{4}{*}{1.0} & \multirow{4}{*}{1.3} & \multirow{4}{*}{1.0} & C & 0.05362 & 0.00473 & 0.09169 & -0.10734 \\
\hline & & & & $P$ & & 0.22330 & 0.04694 & -0.05256 \\
\hline & & & & $r$ & & & 0.36304 & -0.27708 \\
\hline & & & & $\theta$ & & & & 0.33362 \\
\hline \multirow{4}{*}{1.0} & \multirow{4}{*}{1.0} & \multirow{4}{*}{1.5} & \multirow{4}{*}{1.0} & $C$ & 0.05225 & 0.00622 & 0.13867 & -0.10922 \\
\hline & & & & $P$ & & 0.26782 & 0.07868 & -0.06110 \\
\hline & & & & $r$ & & & 0.74628 & -0.44309 \\
\hline & & & & $\theta$ & & & & 0.36300 \\
\hline \multirow{4}{*}{1.0} & \multirow{4}{*}{1.1} & \multirow{4}{*}{1.4} & \multirow{4}{*}{1.0} & C & 0.03979 & -0.00515 & 0.06411 & -0.06696 \\
\hline & & & & $P$ & & 0.25300 & 0.03287 & -0.03543 \\
\hline & & & & $r$ & & & 0.32636 & -0.19642 \\
\hline & & & & $\theta$ & & & & 0.22237 \\
\hline \multirow{4}{*}{1.25} & \multirow{4}{*}{1.1} & \multirow{4}{*}{1.25} & \multirow{4}{*}{1.0} & C & 0.10822 & -0.00295 & 0.12193 & -0.18224 \\
\hline & & & & $P$ & & 0.17555 & 0.18702 & -0.03295 \\
\hline & & & & $r$ & & & 0.28023 & -0.27304 \\
\hline & & & & $\theta$ & & & & 0.42254 \\
\hline \multirow{4}{*}{1.4} & \multirow{4}{*}{1.0} & \multirow{4}{*}{1.0} & \multirow{4}{*}{0.7} & C & 0.09833 & -0.03150 & 0.04490 & -0.07491 \\
\hline & & & & $P$ & & 0.15330 & -0.00493 & 0.00718 \\
\hline & & & & $r$ & & & 0.09398 & -0.05331 \\
\hline & & & & $\theta$ & & & & 0.09605 \\
\hline \multirow{4}{*}{1.4} & \multirow{4}{*}{1.2} & \multirow{4}{*}{1.0} & \multirow{4}{*}{0.9} & $C$ & 0.25885 & -0.03597 & 0.13991 & -0.35472 \\
\hline & & & & $P$ & & 0.14056 & -0.00693 & 0.01586 \\
\hline & & & & $r$ & & & 0.11485 & -0.14831 \\
\hline & & & & $\theta$ & & & & 0.37572 \\
\hline
\end{tabular}

of the stress change time $T_{1}, T_{2}$, say $T_{1}^{*}, T_{2}^{*}$, and the Generalized Asymptotic Variance (GAV) are computed. The numerical results are placed in Tables 5, 6, 7, 8, and 9, which are also based on $n_{1}=29, n_{2}=10, T_{1}=4, T_{2}=3, V_{1}=0.75, V_{2}=1.5$, and $V_{u}=0.5$.

As seen, the results show the optimum values of $T_{1}^{*}, T_{2}^{*}$, and GAV at different values of $\left(C_{0}, P_{0}, \gamma_{0}, \theta_{0}\right)$ and different values of $N$. In general, we observe that GAV decreases as the sample size increases.

\section{Conclusion}

The GL distribution has been extensively used in many different areas and it is very useful in a wide variety of applications, especially in the analysis of survival data. In addition, it is used as the name for several different families of probability distribution. This paper presented the Maximum Likelihood method of the parameter estimation with type-I censoring. The data failure times at each stress level are assumed to follow the 3-parameter-generalized logistic distribution with scale parameter that is an inverse power law function. The ML estimation, Fisher's information matrix, the asymptomatic variance-covariance matrix, the prediction 
Table 4: Estimates $\alpha$ and $R\left(x_{0}\right)$ under normal conditions.

\begin{tabular}{|c|c|c|c|c|c|c|}
\hline$C_{0}$ & $P_{0}$ & $\gamma_{0}$ & $\theta_{0}$ & $\widehat{\alpha}_{u}$ & $x_{0}$ & $\widehat{R}_{u}\left(x_{0}\right)$ \\
\hline \multirow{4}{*}{1.0} & \multirow{4}{*}{1.0} & \multirow{4}{*}{1.25} & \multirow{4}{*}{0.7} & \multirow{4}{*}{1.18740} & 0.01 & 0.43982 \\
\hline & & & & & 0.70 & 0.17726 \\
\hline & & & & & 1.40 & 0.05484 \\
\hline & & & & & 2.00 & 0.01850 \\
\hline \multirow{4}{*}{1.0} & \multirow{4}{*}{1.0} & \multirow{4}{*}{1.3} & \multirow{4}{*}{1.0} & \multirow{4}{*}{1.87408} & 0.02 & 0.4256 \\
\hline & & & & & 0.40 & 0.26659 \\
\hline & & & & & 1.20 & 0.07507 \\
\hline & & & & & 2.00 & 0.00705 \\
\hline \multirow{4}{*}{1.0} & \multirow{4}{*}{1.0} & \multirow{4}{*}{1.5} & \multirow{4}{*}{1.0} & \multirow{4}{*}{1.87408} & 0.005 & 0.39775 \\
\hline & & & & & 0.01 & 0.39551 \\
\hline & & & & & 1.00 & 0.09283 \\
\hline & & & & & 2.00 & 0.01546 \\
\hline \multirow{4}{*}{1.0} & \multirow{4}{*}{1.1} & \multirow{4}{*}{1.4} & \multirow{4}{*}{1.0} & \multirow{4}{*}{1.99560} & 0.02 & 0.40700 \\
\hline & & & & & 0.40 & 0.24330 \\
\hline & & & & & 1.20 & 0.06116 \\
\hline & & & & & 2.50 & 0.00484 \\
\hline \multirow{4}{*}{1.25} & \multirow{4}{*}{1.1} & \multirow{4}{*}{1.25} & \multirow{4}{*}{1.0} & \multirow{4}{*}{2.49450} & 0.02 & 0.43216 \\
\hline & & & & & 0.40 & 0.22777 \\
\hline & & & & & 1.20 & 0.03855 \\
\hline & & & & & 2.50 & 0.00156 \\
\hline \multirow{4}{*}{1.4} & \multirow{4}{*}{1.0} & \multirow{4}{*}{1.0} & \multirow{4}{*}{0.7} & \multirow{4}{*}{2.62371} & 0.02 & 0.52574 \\
\hline & & & & & 0.40 & 0.32054 \\
\hline & & & & & 1.20 & 0.08422 \\
\hline & & & & & 2.50 & 0.00789 \\
\hline \multirow{4}{*}{1.4} & & & & & 0.10 & 0.43900 \\
\hline & 1.2 & 1.0 & 0.9 & 2.97490 & 0.90 & 0.07742 \\
\hline & 1.2 & 1.0 & 0.9 & $2.9 / 490$ & 1.50 & 0.01624 \\
\hline & & & & & 2.00 & 0.00429 \\
\hline
\end{tabular}

Table 5: The optimum values of $T_{1}^{*}, T_{2}^{*}$, and GAV at $\left(C_{0}=1, P_{0}=1, \gamma_{0}=1.5, \theta_{0}=1\right)$.

\begin{tabular}{lccccc}
\hline$N$ & $T_{1}$ & $T_{2}$ & $T_{1}^{*}$ & $T_{2}^{*}$ & GAV \\
\hline 100 & 3 & 4 & 0.124 & 0.116 & 0.00000528 \\
200 & 2 & 3 & 0.292 & 0.332 & 0.00000022 \\
300 & 3 & 8 & 0.074 & 0.237 & 0.00000009 \\
400 & 2 & 7 & 0.238 & 0.893 & 0.00000004 \\
500 & 3 & 7 & 0.129 & 0.260 & 0.00000001 \\
\hline
\end{tabular}

of the value of the scale parameter, and the reliability function under the usual conditions stress were obtained for various combinations of the model parameters. In additional, the corresponding optimum value of the stress change time is obtained numerically by the Doptimality criterion. Since standard Logistic, four-parameter-extended GL, four-parameterextended GL type-I, two-parameter GL, type-I GL, Generalized Log-logistic, standard Loglogistic, Logistic Exponential, Exponentiated Exponential (for $x>0$ ), Generalized Burr, Burr III, and Burr XII distributions are special cases from the GL distribution, then their results of the MLE and optimum test plan become particular cases of the results obtained here. 
Table 6: The optimum values of $T_{1}^{*}, T_{2}^{*}$, and GAV at $\left(C_{0}=1.1, P_{0}=1, \gamma_{0}=1.3, \theta_{0}=1\right)$.

\begin{tabular}{lccccc}
\hline$N$ & $T_{1}$ & $T_{2}$ & $T_{1}^{*}$ & $T_{2}^{*}$ & GAV \\
\hline 100 & 3 & 4 & 0.093 & 0.216 & 0.000002934 \\
200 & 3 & 3 & 0.664 & 0.616 & 0.000000118 \\
300 & 2 & 4 & 1.713 & 1.911 & 0.000000021 \\
400 & 3 & 6 & 0.129 & 0.189 & 0.000000007 \\
500 & 3 & 8 & 0.154 & 0.215 & 0.000000005 \\
\hline
\end{tabular}

Table 7: The optimum values of $T_{1}^{*}, T_{2}^{*}$, and GAV at $\left(C_{0}=1.25, P_{0}=1.2, \gamma_{0}=1.2, \theta_{0}=1\right)$.

\begin{tabular}{lccccc}
\hline$N$ & $T_{1}$ & $T_{2}$ & $T_{1}^{*}$ & $T_{2}^{*}$ & GAV \\
\hline 100 & 3 & 3 & 0.114 & 0.625 & 0.000001655 \\
200 & 2 & 8 & 0.087 & 0.142 & 0.000000312 \\
300 & 2 & 7 & 0.073 & 0.140 & 0.000000109 \\
400 & 3 & 7 & 0.014 & 0.077 & 0.000000022 \\
500 & 2 & 5 & 0.981 & 1.356 & 0.000000003 \\
\hline
\end{tabular}

Table 8: The optimum values of $T_{1}^{*}, T_{2}^{*}$, and GAV at $\left(C_{0}=1.2, P_{0}=1.2, \gamma_{0}=1.5, \theta_{0}=1.2\right)$.

\begin{tabular}{lccccc}
\hline$N$ & $T_{1}$ & $T_{2}$ & $T_{1}^{*}$ & $T_{2}^{*}$ & GAV \\
\hline 100 & 1 & 4 & 0.681 & 1.459 & 0.000007026 \\
200 & 1 & 2 & 1.683 & 1.662 & 0.000000567 \\
300 & 2 & 5 & 0.320 & 1.235 & 0.000000059 \\
400 & 1 & 5 & 0.668 & 1.412 & 0.000000017 \\
500 & 2 & 6 & 0.554 & 1.19 & 0.000000013 \\
\hline
\end{tabular}

Table 9: The optimum values of $T_{1}^{*}, T_{2}^{*}$, and GAV at $\left(C_{0}=1, P_{0}=1.1, \gamma_{0}=1.4, \theta_{0}=1\right)$.

\begin{tabular}{lccccc}
\hline$N$ & $T_{1}$ & $T_{2}$ & $T_{1}^{*}$ & $T_{2}^{*}$ & GAV \\
\hline 100 & 3 & 4 & 0.090 & 0.024 & 0.000002541 \\
200 & 2 & 3 & 0.263 & 0.192 & 0.000000119 \\
300 & 3 & 3 & 0.088 & 0.112 & 0.000000409 \\
400 & 3 & 5 & 0.336 & 0.399 & 0.000000006 \\
500 & 3 & 8 & 0.025 & 0.065 & 0.000000003 \\
\hline
\end{tabular}

\section{Appendix}

The determinant of $F$ is

$$
\begin{aligned}
|F|= & \left(f_{33} f_{44}-f_{34}^{2}\right)\left(f_{11} f_{22}-f_{12}^{2}\right)-\left(f_{23} f_{44}-f_{24} f_{34}\right)\left(f_{11} f_{23}-f_{12} f_{13}\right) \\
& +\left(f_{23} f_{34}-f_{24} f_{33}\right)\left(f_{11} f_{24}-f_{12} f_{14}\right)-\left(f_{13} f_{44}-f_{14} f_{34}\right)\left(f_{13} f_{22}-f_{12} f_{23}\right) \\
& +\left(f_{13} f_{34}-f_{33} f_{14}\right)\left(f_{14} f_{22}-f_{12} f_{24}\right)-\left(f_{13} f_{24}-f_{23} f_{14}\right)\left(f_{14} f_{23}-f_{13} f_{24}\right) .
\end{aligned}
$$


The derivative of $|F|$ for obtaining the optimum value of the censoring time $T_{j}, j=$ $1, \ldots, k$ is given as follows:

$$
\begin{aligned}
\frac{\partial|F|}{\partial T_{j}}= & \left(f_{33} f_{44}-f_{34}^{2}\right)\left(f_{11}^{\prime} f_{22}+f_{11} f_{22}^{\prime}-2 f_{12} f_{12}^{\prime}\right) \\
& +\left(f_{33}^{\prime} f_{44}+f_{33} f_{44}^{\prime}-2 f_{34}^{\prime} f_{34}\right)\left(f_{11} f_{22}-f_{12}^{2}\right) \\
& -\left(f_{23}^{\prime} f_{44}+f_{23} f_{44}^{\prime}-f_{24}^{\prime} f_{34}-f_{24} f_{34}^{\prime}\right)\left(f_{11} f_{23}-f_{12} f_{13}\right) \\
& -\left(f_{23} f_{44}-f_{24} f_{34}\right)\left(f_{11}^{\prime} f_{23}+f_{11} f_{23}^{\prime}-f_{12}^{\prime} f_{13}-f_{12} f_{13}^{\prime}\right) \\
& +\left(f_{23} f_{34}-f_{24} f_{33}\right)\left(f_{11}^{\prime} f_{24}+f_{11} f_{24}^{\prime}-f_{12}^{\prime} f_{14}-f_{12} f_{14}^{\prime}\right) \\
& +\left(f_{23}^{\prime} f_{34}+f_{23} f_{34}^{\prime}-f_{24}^{\prime} f_{33}-f_{24} f_{33}^{\prime}\right)\left(f_{11} f_{24}-f_{12} f_{14}\right) \\
& -\left(f_{13} f_{44}-f_{14} f_{34}\right)\left(f_{13}^{\prime} f_{22}+f_{13} f_{22}^{\prime}-f_{12}^{\prime} f_{23}-f_{12} f_{23}^{\prime}\right) \\
& -\left(f_{13}^{\prime} f_{44}+f_{13} f_{44}^{\prime}-f_{14}^{\prime} f_{34}-f_{14} f_{34}^{\prime}\right)\left(f_{13} f_{22}-f_{12} f_{23}\right) \\
& +\left(f_{13} f_{34}-f_{33} f_{14}\right)\left(f_{14}^{\prime} f_{22}+f_{14} f_{22}^{\prime}-f_{12}^{\prime} f_{24}-f_{12} f_{24}^{\prime}\right) \\
& +\left(f_{13}^{\prime} f_{34}+f_{13} f_{34}^{\prime}-f_{33}^{\prime} f_{14}-f_{33} f_{14}^{\prime}\right)\left(f_{14} f_{22}-f_{12} f_{24}\right) \\
& -\left(f_{13} f_{24}-f_{23} f_{14}\right)\left(f_{14}^{\prime} f_{23}+f_{14} f_{23}^{\prime}-f_{13}^{\prime} f_{24}-f_{13} f_{24}^{\prime}\right) \\
& -\left(f_{13}^{\prime} f_{24}+f_{13} f_{24}^{\prime}-f_{23}^{\prime} f_{14}-f_{23} f_{14}^{\prime}\right)\left(f_{14} f_{23}-f_{13} f_{24}\right),
\end{aligned}
$$

where

$$
\begin{gathered}
f_{11}^{\prime}=\frac{\theta^{2}}{r} \sum_{i=1}^{n_{j}}\left(1-\delta_{i j}\right) S_{j}{ }^{P} Z_{j}\left(1-\mu_{j}\right)\left[C S_{j}{ }^{P} T_{j}\left(1-2 \mu_{j}\right)+2\right], \\
f_{22}^{\prime}=C \theta \sum_{i=1}^{n_{j}}\left(1-\delta_{i j}\right) S_{j}^{P}\left(\ln S_{j}\right)^{2} \mu_{j}\left[C S_{j}{ }^{P} T_{j}\left(1-\mu_{j}\right)\left(1-C Z_{j}\right)+\left(1+C S_{j}{ }^{P} T_{j}\left(1-\mu_{j}\right)\right)^{2}\right], \\
f_{33}^{\prime}=\frac{-2 C \theta}{r^{2}} \sum_{i=1}^{n_{j}}\left(1-\delta_{i j}\right) S_{j}{ }^{P}\left(1-\mu_{j}\right) \mu_{j}^{2} \\
f_{44}^{\prime}=\frac{-2 C}{\theta} \sum_{i=1}^{n_{j}}\left(1-\delta_{i j}\right) S_{j}{ }^{P}\left(1-\mu_{j}\right) \mu_{j}^{2} \\
f_{12}^{\prime}=\theta \sum_{i=1}^{n_{j}}\left(1-\delta_{i j}\right) S_{j}^{P} \ln S_{j} \mu_{j}\left[C S_{j}{ }^{P} T_{j}\left(1-\mu_{j}\right)\left(1-C Z_{j}\right)+\left(1+C S_{j}{ }^{P} T_{j}\left(1-\mu_{j}\right)\right)^{2}\right], \\
f_{13}^{\prime}=\frac{\theta}{r} \sum_{i=1}^{n_{j}}\left(1-\delta_{i j}\right) S_{j}^{P} \mu_{j}\left(1-\mu_{j}\right)\left[1+C S_{j}{ }^{P} T_{j}\left(1-2 \mu_{j}\right)\right],
\end{gathered}
$$




$$
\begin{gathered}
f_{14}^{\prime}=\sum_{i=1}^{n_{j}}\left(1-\delta_{i j}\right) S_{j}^{P} \mu_{j}{ }^{2}\left(1+2 C S_{j}{ }^{P} T_{j}\left(1-\mu_{j}\right)\right), \\
f_{23}^{\prime}=\frac{C \theta}{r} \sum_{i=1}^{n_{j}}\left(1-\delta_{i j}\right) S_{j}^{P} \ln S_{j} \mu_{j}\left(1-\mu_{j}\right)\left[1+C S_{j}{ }^{P} T_{j}\left(1-2 \mu_{j}\right)\right], \\
f_{24}^{\prime}=C \sum_{i=1}^{n_{j}}\left(1-\delta_{i j}\right) S_{j}^{P} \ln S_{j} \mu_{j}{ }^{2}\left[1+2 C S_{j}{ }^{P} T_{j}\left(1-\mu_{j}\right)\right], \\
f_{34}^{\prime}=\frac{2 C}{r} \sum_{i=1}^{n_{j}}\left(1-\delta_{i j}\right) S_{j}^{P} \mu_{j}^{2}\left(1-\mu_{j}\right) .
\end{gathered}
$$

\section{References}

[1] J. F. Lawless, "Confidence interval estimation in the inverse power law model," Journal of the Royal Statistical Society: Series C, vol. 25, no. 2, pp. 128-138, 1976.

[2] J. I. McCool, "Confidence limits for weibull regression with censored data," IEEE Transactions on Reliability, vol. R-29, no. 2, pp. 145-150, 1980.

[3] D. S. Bai and S. W. Chung, "An accelerated life test model with the inverse power law," Reliability Engineering and System Safety, vol. 24, no. 3, pp. 223-230, 1989.

[4] M. M. Bugaighis, "Properties of the MLE for parameters of a Weibull regression model under type I censoring," IEEE Transactions on Reliability, vol. 39, no. 1, pp. 102-105, 1990.

[5] A. J. Watkins, "On the analysis of accelerated life-testing experiments," IEEE Transactions on Reliability, vol. 40, no. 1, pp. 98-101, 1991.

[6] A. A. Abdel-Ghaly, A. F. Attia, and H. M. Aly, "Estimation of the parameters of pareto distribution and the reliability function using accelerated life testing with censoring," Communications in Statistics Part B, vol. 27, no. 2, pp. 469-484, 1998.

[7] E. A. El-Dessouky, On the use of bayesian approach in accelerated life testing, M.S. thesis, Institute of Statistical Studies and Research, Cairo University, Egypt, 2001.

[8] W. Q. Meeker, L. A. Escobar, and C. J. Lu, "Accelerated degradation tests: modeling and analysis," Technometrics, vol. 40, no. 2, pp. 89-99, 1998.

[9] W. Nelson and T. J. Kielpinski, "Theory for optimum censored accelerated life tests for normal and lognormal life distributions," Technometrics, vol. 18, no. 1, pp. 105-114, 1976.

[10] W. Nelson, Accelerated Life Testing: Statistical Models, Test Plan and Data Analysis, John Wiley \& Sons, New York, NY, USA, 1990.

[11] G. B. Yang, "Optimum constant-stress accelerated life-test plans," IEEE Transactions on Reliability, vol. 43, no. 4, pp. 575-581, 1994.

[12] C. Ding, C. Yang, and S. K. Tse, "Accelerated life test sampling plans for the Weibull distribution under type I progressive interval censoring with random removals," Journal of Statistical Computation and Simulation, vol. 80, no. 8, pp. 903-914, 2010.

[13] N. L. Johnson, S. Kotz, and N. Balakrishnan, Continuous Univariate Distributions, vol. 2 of Wiley Series in Probability and Mathematical Statistics: Applied Probability and Statistics, John Wiley \& Sons, New York, NY, USA, 2nd edition, 1995.

[14] S. Nadarajah and S. Kotz, "A generalized logistic distribution," International Journal of Mathematics and Mathematical Sciences, vol. 2005, no. 19, pp. 3169-3174, 2005.

[15] A. M. Mathai and S. B. Provost, "On the distribution of order statistics from generalized logistic samples," International Journal of Statistics, vol. 62, no. 1, pp. 63-71, 2004.

[16] K. Tolikas, "Value-at-risk and extreme value distributions for financial returns," Journal of Risk, vol. 10, pp. 31-77, 2008.

[17] M. R. Alkasasbeh and M. Z. Raqab, "Estimation of the generalized logistic distribution parameters: comparative study," Statistical Methodology, vol. 6, no. 3, pp. 262-279, 2009. 
[18] K. Tolikas and G. D. Gettinby, "Modelling the distribution of the extreme share returns in Singapore," Journal of Empirical Finance, vol. 16, no. 2, pp. 254-263, 2009.

[19] A. Shabri, U. N. Ahmad, Z. A. Zakaria et al., "TL-moments and L-moments estimation of the generalized logistic distribution," Journal of Mathematical Research, vol. 10, no. 10, pp. 97-106, 2011.

[20] K. Tolikas, "The rare event risk in African emerging stock markets," Managerial Finance, vol. 37, no. 3, pp. 275-294, 2011.

[21] H. Shin, T. Kim, S. Kim, and J. H. Heo, "Estimation of asymptotic variances of quantiles for the generalized logistic distribution," Stochastic Environmental Research and Risk Assessment, vol. 24, no. 2, pp. 183-197, 2010.

[22] R. K. Rai, S. Sarkar, and V. P. Singh, "Evaluation of the adequacy of statistical distribution functions for deriving unit hydrograph," Water Resources Management, vol. 23, no. 5, pp. 899-929, 2009.

[23] S. Kim, H. Shin, T. Kim, and J. Heo, "Derivation of the probability plot correlation coefficient test statistics for the generalized logistic distribution," in Proceedings of the International Workshop Advances in Statistical Hydrology, Taormina, Italy, 2010.

[24] G. Molenberghs and G. Verbeke, "On the Weibull-Gamma frailty model, its infinite moments, and its connection to generalized log-logistic, logistic, Cauchy, and extreme-value distributions," Journal of Statistical Planning and Inference, vol. 14, no. 2, pp. 861-868, 2011.

[25] E. Gouno, "Optimum step-stress for temperature accelerated life testing," Quality and Reliability Engineering International, vol. 23, no. 8, pp. 915-924, 2007. 


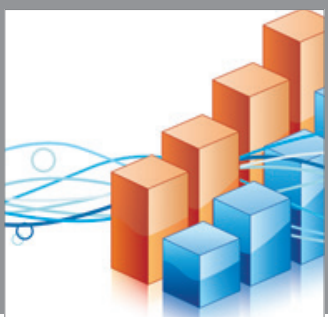

Advances in

Operations Research

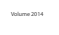

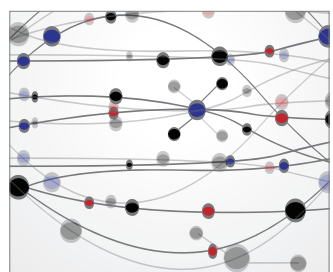

\section{The Scientific} World Journal
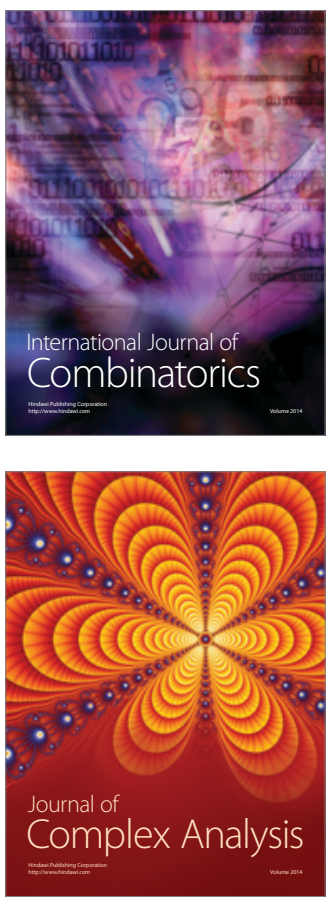

International Journal of

Mathematics and

Mathematical

Sciences
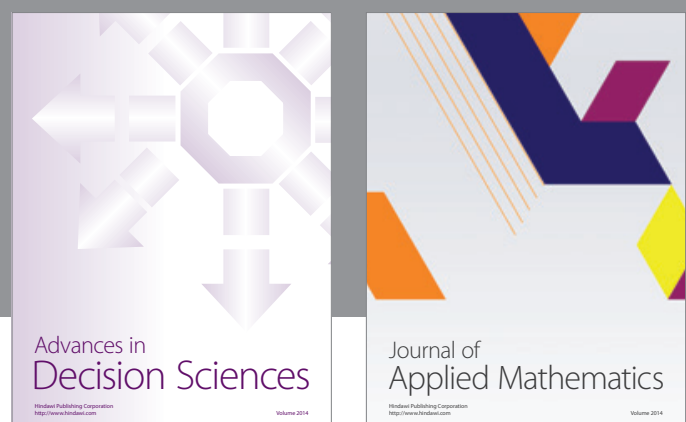

Journal of

Applied Mathematics
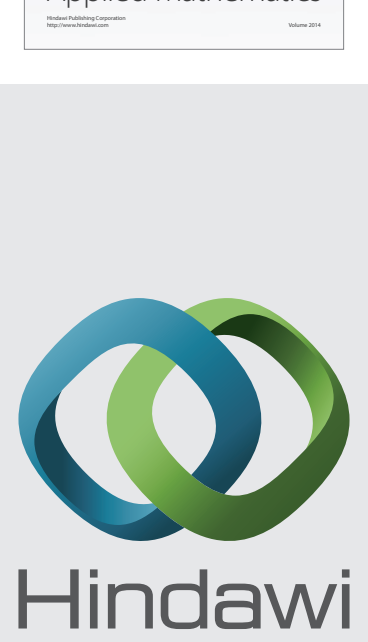

Submit your manuscripts at http://www.hindawi.com
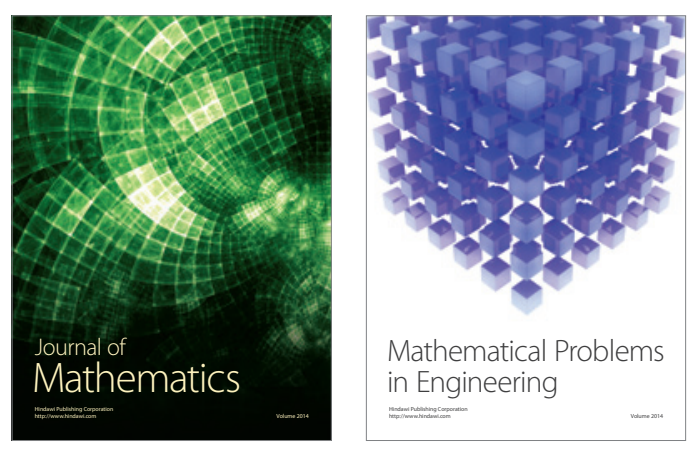

Mathematical Problems in Engineering
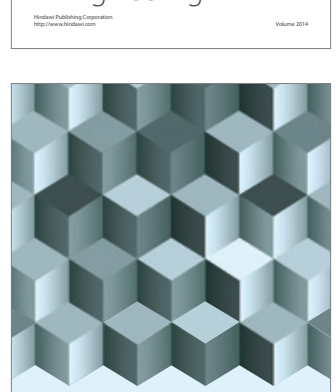

Journal of

Function Spaces
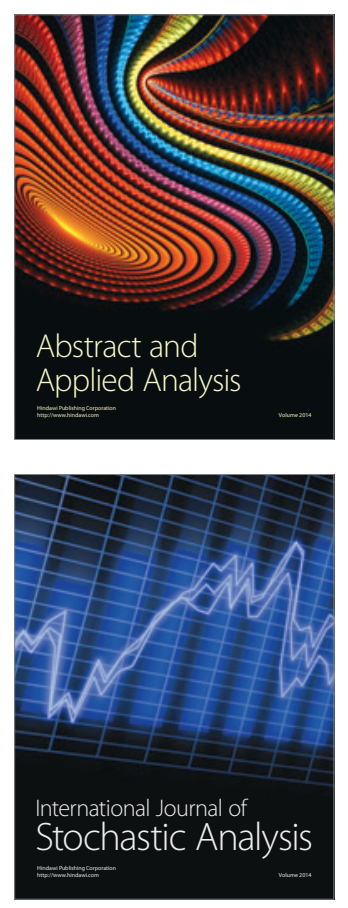

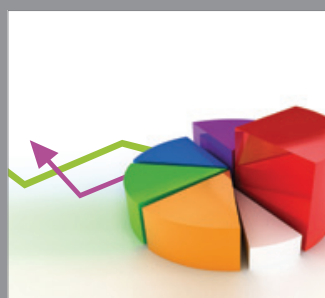

ournal of

Probability and Statistics

Promensencen
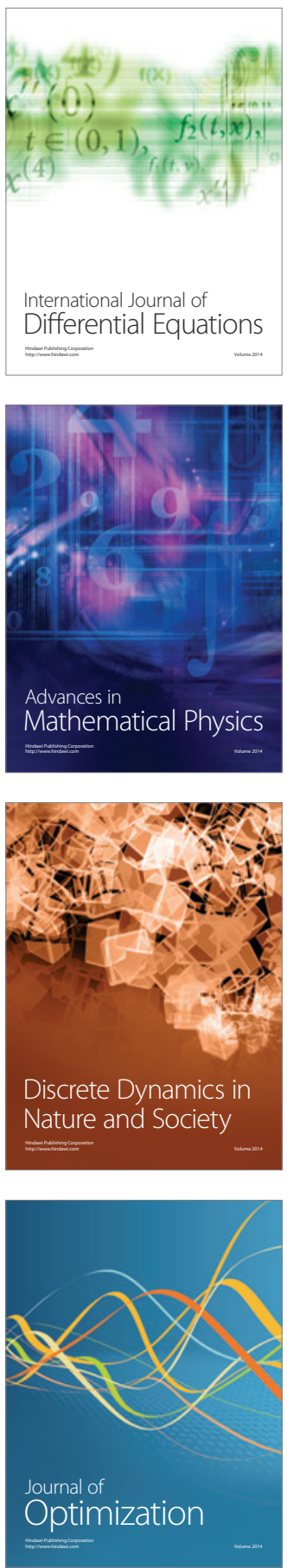